\title{
A surgical approach to coexistent coronary and carotid artery disease
}

\author{
Marjan Jahangiri, Gareth M Rees, Stephen J Edmondson, John Lumley, Rakesh Uppal
}

\begin{abstract}
Objective-To assess the early results of combined coronary artery bypass graft surgery and carotid endarterectomy. Design-Retrospective and ongoing analysis of patients who underwent combined coronary artery bypass graft surgery and carotid endarterectomy. Setting - Cardiothoracic unit in a London teaching hospital.

Patients-From June 1987 to March 1995, 64 patients were identified. They were patients who were scheduled to have coronary artery bypass graft surgery or required urgent coronary revascularisation and who were found to have significant coexistent carotid disease. (Unilateral carotid stenosis $>70 \%$, bilateral carotid stenosis $>50 \%$, or unilateral carotid stenosis $>\mathbf{5 0} \%$ with contralateral occlusion.)
\end{abstract}

Interventions-Both procedures were performed during one anaesthesia: the carotid endarterectomy was performed first without cardiopulmonary bypass. After completion of carotid endarterectomy, coronary artery bypass graft surgery was performed.

Main outcome measures-The incidence of stroke, transient ischaemic attack, and myocardial infarction in the early postoperative period was analysed.

Results-Myocardial revascularisation was successful in all 64 patients. There were no perioperative infarcts. In three patients (4.7\%) a new neurological deficit developed postoperatively: two recovered fully before hospital discharge.

Conclusions-Combined coronary artery bypass graft surgery and carotid endarterectomy were performed safely and with good results.

(Heart 1997;77:164-167)

Keywords: coronary artery bypass graft surgery; carotid endarterectomy; stroke; myocardial infarction

The management of coexistent coronary artery and carotid artery disease is controversial. This was highlighted in a recent editorial in the British Heart fournal. ${ }^{1}$ Carotid endarterectomy in patients with significant coronary artery disease is associated with a perioperative myocardial infarct rate of up to $15 \%$. It has also been shown that the greatest long term risk to all patients who have carotid endarterectomy is posed by the complications of coronary disease. ${ }^{23}$ Similarly, coronary artery bypass graft surgery in the presence of significant carotid disease is associated with a $15 \%$ risk of perioperative stroke. ${ }^{4} \mathrm{We}$ set out to investigate whether simultaneous combined coronary artery bypass graft surgery and carotid endarterectomy decrease these risks. Several studies have shown an association between history of stroke or transient ischaemic attack and carotid stenosis. ${ }^{5}$ Recently in a prospective randomised study of 470 men aged 68 years, the incidence of stroke, transient ischaemic attack, and all cause mortality during 10 years of follow up in relation to carotid stenosis was studied. The investigators failed to show an association between carotid stenosis and increased risk of stroke. Part of this lack of association was explained by the high mortality from ischaemic heart disease in men with severe stenosis. $^{6}$

We report our experience of combined simultaneous coronary artery bypass graft surgery and carotid endarterectomy. A review of the pertinent literature on patients with symptomatic and asymptomatic carotid disease undergoing coronary artery bypass graft surgery is presented.

\section{Methods}

A retrospective and ongoing analysis of patients who underwent combined carotid endarterectomy and coronary artery bypass graft surgery was carried out. The study population consisted of patients who were scheduled to have elective coronary artery bypass graft surgery or required emergency bypass operation and were found to have significant carotid disease. From June 1987 to March 1995, 64 patients were identified. Twenty nine $(45.3 \%)$ of these patients were operated on in the last two years of the study. The mean age was $65 \cdot 2$ years (range 54-73) with a male to female ratio of $1 \cdot 5: 1$. Fifty one patients $(80 \%)$ had three vessel coronary artery disease and 13 $(20 \%)$ had two vessel disease. Twelve $(18 \cdot 8 \%)$ had unstable angina, $13(20.3 \%)$ had moderately impaired left ventricular function, and 11 $(17 \cdot 1 \%)$ had over $50 \%$ stenosis of the left main coronary artery.

Forty nine patients $(76 \cdot 6 \%)$ had a carotid bruit and $39(60 \%)$ had a history of transient ischaemic attack or stroke. All patients were investigated with Doppler ultrasound. A lesion was considered haemodynamically significant when the stenosis was $>70 \%$ of the luminal 
diameter in both the symptomatic and asymptomatic patients. Patients with a significant carotid lesion were referred to a vascular surgeon. Carotid angiography was subsequently performed in 52 patients $(81.3 \%)$ which confirmed the Doppler findings. Nine patients $(14.1 \%)$ had bilateral significant carotid disease. In these, the side contributing to the majority of cerebral blood flow was operated on first. Six patients $(9 \cdot 4 \%)$ had unilateral stenosis with contralateral carotid obstruction. In these patients no surgery was performed on the occluded side.

In our unit, carotid endarterectomy and coronary artery bypass graft surgery are performed under one general anaesthesia. Carotid endarterectomy is performed before median sternotomy without cardiopulmonary bypass and without shunting. The endarterectomy incision is closed directly, with no patch. The wound is closed over an appropriate drain. Heparin in given before cannulation or just before division of the internal mammary artery if it is being taken down. The left internal mammary artery is routinely taken down if the coronary anatomy is suitable, irrespective of the carotid disease status. Coronary artery bypass graft surgery is performed with cold antegrade crystalloid cardioplegia and systemic hypothermia at $28^{\circ} \mathrm{C}$. During cardiopulmonary bypass the perfusion pressure is maintained at $55-80 \mathrm{~mm} \mathrm{Hg}$.

\section{Results}

There were no perioperative deaths. Three patients $(4 \cdot 7 \%)$ developed a new neurological deficit postoperatively, two of whom recovered fully before discharge from hospital. One patient who underwent triple coronary artery bypass graft surgery and left internal carotid endarterectomy with a previous history of stroke developed aphasia and right sided arm weakness following surgery. She recovered fully and was discharged home four weeks after surgery. Another patient with no neurological history who underwent left internal carotid endarterectomy developed a transient weakness of his right limbs. Computed tomography of the brain was performed in both patients and showed no significant abnormalities. The third patient had had a stroke four years before presentation affecting his right leg. Following left internal carotid endarterectomy and coronary artery bypass graft surgery the weakness of his leg deteriorated and he was discharged home with no apparent improvement. There were no perioperative myocardial infarcts or major complications. The median time of stay in the hospital following surgery was eight days (range six to 29 days).

\section{Discussion}

The management of patients with coexistent coronary artery and carotid artery disease is controversial and difficult. Many studies suggest that simultaneous combined carotid endarterectomy and coronary artery bypass graft surgery is the safest approach. The risk of perioperative stroke has been shown to be significantly higher in patients undergoing coronary artery bypass graft with carotid stenosis of more than $70 \%$ compared to patients without carotid stenosis. Similarly, a stroke rate of $8.9 \%$ in coronary artery bypass graft patients with angiographically documented carotid stenosis ( $>70 \%)$ has been reported, compared to $1.3 \%$ in patients without carotid stenosis. ${ }^{7}$

Here we discuss asymptomatic and symptomatic carotid disease in association with coronary artery disease.

\section{ASYMPTOMATIC DISEASE}

In the current series $40 \%$ of patients were asymptomatic, that is, they did not have a history of stroke or transient ischaemic attacks. One patient $(4 \cdot 1 \%)$ developed a transient neurological deficit. This is a lower incidence than in some reported series. A $\mathbf{9 \cdot 2 \%}$ incidence of transient ischaemic attack or stroke has been reported in coronary artery bypass graft patients with carotid stenosis, as compared to $1.9 \%$ risk in patients without carotid stenosis. ${ }^{8}$ Hobson and colleagues reported a significant reduction in the overall incidence of ipsilateral neurological events after carotid endarterectomy in patients with asymptomatic lesions. ${ }^{9} \mathrm{~A}$ review of published reports indicates that all series reported on combined procedures are retrospective analyses except one prospective randomised trial reported by Hertzer et al. ${ }^{7}$ In a prospective trial, 129 patients with unilateral asymptomatic severe carotid stenosis were randomised to either combined carotid endarterectomy and coronary artery bypass graft performed simultaneously $(n=71)$ or coronary artery bypass graft followed by carotid endarterectomy ("reverse staged", $\mathbf{n}=$ 58) during convalescence in the hospital or at a later date. The mortality in patients who underwent a combined operation was $4 \cdot 2 \%$, with a stroke rate of $2 \cdot 8 \%$, as opposed to a stroke rate of $14 \%(P=0.042)$ with a mortality of $5.2 \%$ in the reverse staged group. The asymptomatic carotid atherosclerosis study (ACAS) showed that in patients with asymptomatic carotid stenosis of $60 \%$ or more, elective carotid endarterectomy will have a five year reduced risk of ipsilateral stroke. ${ }^{10}$ More recently, the National Institutes of Health abruptly ended the randomised trial of carotid endarterectomy versus medical treatment in patients with asymptomatic carotid disease because of the very significant advantage of surgery. ${ }^{11}$

\section{SYMPTOMATIC DISEASE}

For symptomatic patients, carotid endarterectomy provided superior stroke prevention in the presence of unilateral stenosis of more than $70 \%$, bilateral stenosis of more than $50 \%$, and internal carotid occlusion associated with more than $50 \%$ contralateral stenosis. ${ }^{12}$ These findings are confirmed by the North American symptomatic carotid endarterectomy trial (NASCET) ${ }^{13}$ and the European carotid surgery trial. ${ }^{14}$ From the Cleveland series, ${ }^{7}$ for the management of symptomatic 
carotid disease, it was considered unethical to randomise the patients to combined carotid endarterectomy and coronary artery bypass graft surgery or coronary artery bypass graft followed by carotid endarterectomy because their risk for neurological complications seemed higher than in those with asymptomatic disease. Ninety nine patients underwent combined procedure, with a mortality rate of $6 \cdot 6 \%$ and a stroke rate of $7 \cdot 1 \%$. The mortality and stroke rates in symptomatic patients are higher than in those with asymptomatic disease. This could partly be due to the increased incidence of atherosclerotic risk factors such as hypertension, diabetes, and a history of smoking in the symptomatic group as opposed to the asymptomatic group. Several studies have reported a higher incidence of left main coronary disease in patients with a history of transient ischaemic attack or stroke as compared to the asymptomatic patients with significant carotid stenosis. We found no significant difference in the incidence of left main coronary disease between the symptomatic and the asymptomatic group. This incidence is also higher in patients undergoing combined procedure compared with those undergoing coronary artery bypass graft surgery. The reason remains unexplained. ${ }^{1516}$

The timing of carotid endarterectomy in relation to coronary artery bypass graft surgery is an important issue. We believe that because of the increased risk of stroke in the presence of coexistent disease, combined simultaneous carotid endarterectomy and coronary artery bypass graft surgery should be the option of choice. Another option would be to perform carotid endarterectomy sometime before coronary artery bypass graft surgery. Twenty four out of 275 patients in the Cleveland series had carotid endarterectomy performed sometime before coronary artery bypass graft and they were considered to have "such acceptable cardiac parameters" that staged preliminary carotid endarterectomy could be done safely before coronary artery bypass grafting. One $(4 \cdot 2 \%)$ of the 24 patients sustained an ipsilateral hemispheric stroke after carotid

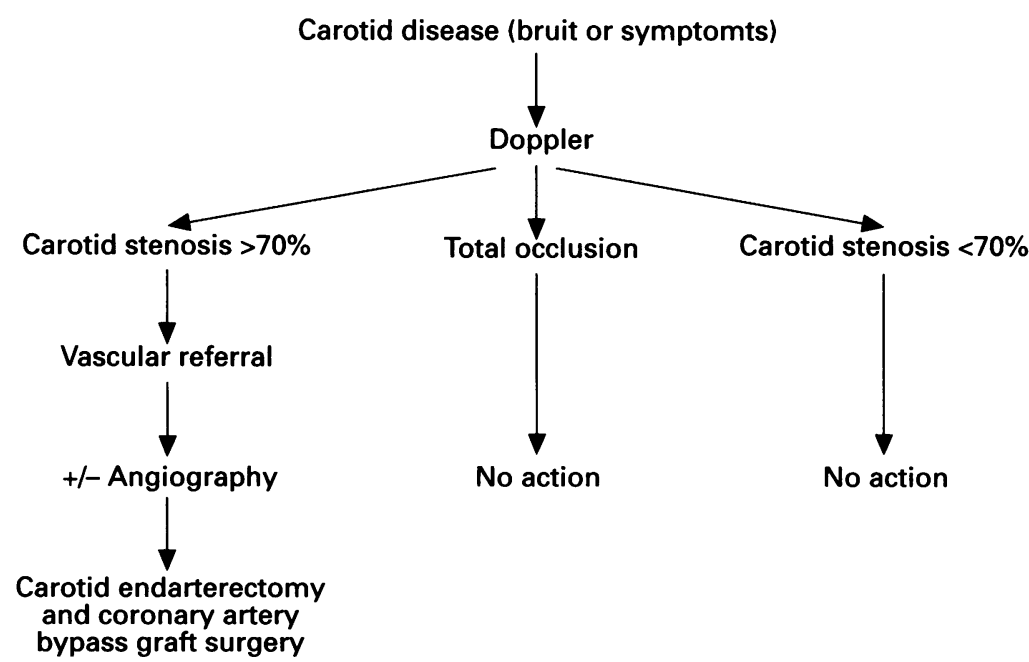

Algorithm for the management of coexistent carotid stenosis in patients undergoing coronary artery bypass graft surgery. endarterectomy and died of a myocardial infarct during the prolonged staging interval. There are no data to specifically define and address these patients with "acceptable cardiac parameters". Perhaps patients with one or two vessel stable coronary artery disease would be appropriate candidates for preliminary carotid endarterectomy followed by coronary artery bypass graft. However, this seems a dangerous option given a myocardial infarct risk of nearly $15 \%$ in the staging interval.

Like most other groups, we apply the two team technique and perform carotid endarterectomy before coronary artery bypass graft surgery without cardiopulmonary bypass. We believe the institution of cardiopulmonary bypass in the presence of a carotid stenosis could cause cerebral hypoperfusion leading to stroke. In practice having two groups of surgeons operating simultaneously can be difficult. The advocates of carotid endarterectomy with cardiopulmonary bypass believe that hypothermia provides some cerebral protection. There are no data to support this in adults.

For the management of bilateral carotid disease we concur with the management of Carrel and colleagues, ${ }^{15}$ that carotid endarterectomy should be performed on the side which contributes to the majority of cerebral blood flow first, and on the other side at a later date after the combined procedure. In the presence of total occlusion, carotid endarterectomy is not performed. A recent report on the outcome of symptomatic carotid disease in young adults suggests that patients with total occlusion have a benign prognosis. ${ }^{17}$ To our knowledge, this is the largest series reported in Europe on the management of coexistent coronary and carotid artery disease.

Forty five per cent of our patients were identified in the last two years of the study. We believe this is because of the increased awareness by our team of this particular problem. We did not introduce a different screening process in the last two years of the study.

We have provisionally constructed an algorithm (figure) for the management of coexistent coronary artery and carotid artery disease. We conclude that in the presence of significant carotid disease in patients undergoing coronary artery bypass graft surgery, combined coronary artery bypass graft and carotid endarterectomy can be performed safely. Combined surgery avoids the costs of a second hospital admission, accelerates treatment of two different diseases, and eliminates the risks associated with the still present disease in the waiting period for the second operation. It is more comfortable for the patient who will have only one episode of anaesthesia and one hospital admission.

Akins CW. The case for concomitant carotid and coronary artery surgery. Br Heart f 1995;74:97-8.

Hertzer NR, Lees CD. Fatal myocardial infarction following carotid endarterectomy. Ann Surg 1981;194:212-8.

3 Hertzer NR, Arison R. Cumulative stroke and survival ten years after carotid endarterectomy. F Vasc Surg 1985;2: 661-8.

4 Faggioli GL, Curl R, Ricotta J. The role of carotid screen ing before coronary artery bypass. $\mathcal{F}$ Vasc Surg 1990;12: 724-31. 
5 Junquist G, Milsson B, Ostbergh A, Isacsson SO, Kanzon L, Steen B, et al. Carotid artery blood flow velocity related to transient ischaemic attack and stroke in a population study of 69 year old men. Stroke 1989;20:1327-30. 6 Ogren M, Hedblad BO, Isacsson SO, Janzon L, Jungquist $\mathrm{G}$, Lindell SE. Ten year old cardiovascular morbidity carotid stenosis. $B M \mathcal{F}$ 1995;310:1294-8.

7 Hertzer NR, Loop FD, Bevan EG, O'Hara PJ, Krajewski LP. Surgical staging of simultaneous coronary and carotid disease A study including prospective randomisation. 7 Vasc Surg 1989;9:455-63.

8 Brenner BT, Brief DK, Alport J, Goldenkrawl RJ, Parsonnet $\mathrm{V}$. The risk of stroke in patients with asymptomatic carotid stenosis undergoing cardiac surgery: a follow up study. F Vasc Surg 1987;5:269-77.

9 Hobson RW, Weiss DG, Fields WS. Efficacy of carotid endarterectomy for asymptomatic carotid stenosis. $N$ endarterectomy for asympto
Engl $₹$ Med 1993;308:201-9.

10 Executive Committee for the Asymptomatic Carotid Atherosclerosis Study. Endarterectomy for asymptomatic carotid artery stenosis. $\mathscr{f} A M A$ 1995;273:1421-8.
11 Altman LK. The New York Times 1994; October 1, p 1.

12 Hertzer NR, Flanagan RA, O'Hara PJ. Surgical versus nonoperative treatment of symptomatic carotid stenosis. $A n n$ Surg 1986;204:154-62.

13 North American Symptomatic Carotid Endarterectomy Trial (NASCET). North American Symptomatic Carotid Endarterectomy Trial: methods, patient characteristics and progress. Stroke 1991;22:711-20.

14 European Carotid Surgery Trialists Collaborative Group. MRC European Carotid Surgery Trial: interim results for symptomatic patients severe $(70 \%-99 \%)$ or mild (0-29\%) carotid stenosis. Lancet 1991;337:1235-43

15 Carrel T, Stillhard G, Turina M. Combined carotid and coronary artery surgery: early and late results. Cardiology 1992;80:118-25.

16 Cosgrove DM, Hertzer NR, Loop FD. Surgical management of synchronous carotid and coronary artery disease. f Vasc Surg 1986;3:690-5.

17 Carolei A, Marini C, Nencini P, Gandolfo C, Molto $\mathrm{C}$, Zenette E, et al. Prevalence and outcome of symptomatic carotid lesions in young adults. $B M F$ 1995;310 1363-6. 